\title{
THE PROCESSING OF ENGLISH WORDS IGNORED IN JAPANESE UNIVERSITY STUDENTS
}

\author{
Satoko IKEDA ${ }^{1)}$ \\ ${ }^{1)}$ Yasuda Women's University, Japan
}

\begin{abstract}
The present study aimed to examine to what extent an English word ignored is processed. The subjects were required to name the category of a target word, ignoring a distracting word. Although the English and Japanese words used in the present study were similar in sound, they were different in script. The results showed that inhibition was produced in categorizing a Japanese target word even when an English word was used as a distracting word. This inhibition was not produced in the previous similar study using ordinary words (Ikeda, 1993). The results of the present study suggested an English word ignored is automatically phonetically processed.
\end{abstract}

Key words: English words, Japanese words, lexical representation, conceptual representation, Japanese university students

In the studies of bilingualism, both the lexical representation in the lexicon and the conceptual representation in the semantic memory were assumed (Potter, So, Von Eckardt, \& Feldman, 1984; Snodgrass, 1984). A word is represented as a lexical representation in the lexicon which is a device in processing a word based on its sound and spelling, on the other hand, as a concept representation in the semantic memory which is a device in processing a word based on its meaning. If the subjects are presented a word and required to process the word semantically, the processing pathway of the word from the lexicon to the semantic memory has to be passed (Glaser \& Glaser, 1989).

Some previous studies of bilingualism examined whether the two languages maintain a single integrated semantic memory (Caramazza \& Brones, 1980; Chen \& Ng, 1989; Guttentag, Haith, Goodman, \& Hauch, 1984; Ikeda, 1993). An example of such a study is that of Guttentag et al. (1984). They presented a target word surrounded above and below by flanker words, and required the subjects to press one of the two keys depending on the category of the target word, ignoring the flanker words. The results showed that the reaction time to the target words was affected by the semantic relationship between the flankers and the targets even when the targets and flankers were written in different languages. The results implied a common semantic memory between the two languages.

Ikeda (1993) examined the semantic processing of English and Japanese words (e.g., リンゴ [apple], キツネ[fox]) which were different in sound and script, using the wordword categorizing task (Stroop-like-task). In this task, the subjects were required to tell the category of a target word, ignoring a distracting word. When the reaction time to the

Correspondence concerning this article should be addressed to Satoko Ikeda, Department of Psychology, Faculty of Letters, Yasuda Women's University, Yasuhigashi, Asaminami-ku, Hiroshima 731-0153, Japan (e-mail: stikeda@yasuda-u.ac.jp). 
target was delayed, it was assumed that inhibition was produced by the distracting word. On the other hand, when the reaction time to the target was accelerated, it was assumed that facilitation was produced by the distracting word. The results of this study showed that the Japanese distracting words produced inhibition and facilitation to the English target words, and the inhibition was affected by the semantic relationship between a target word and a distracting word (Experiment 1). These results implied that English words were processed in the common semantic memory to Japanese words. On the other hand, the English distracting words produced neither inhibition nor facilitation to the Japanese words (Experiment 2). These results implied that English words did not affect the semantic processing of Japanese words.

de Groot and Nas (1991) also examined the representation of a word in the lexicon of Dutch-English bilingual by the priming paradigm. They used cognates and noncognates as targets and primes. Cognates are the words of which the word form in one language is very similar to the word form in the other language both in sound and in spelling. The results of the study indicated that the equality of the associative (semantic)-priming effects within- and between-languages was restricted to cognates, and the between-languages associative-priming effects failed to be exhibited on noncognates.

Though Japanese has no cognates, Japanese has a lot of words of foreign origin (loanwords) which are similar to English words in sound, but different from them in script. The present study used those Japanese and English words in the word-word categorizing task, and compared the results with those of the previous studies using ordinary words (Ikeda, 1993) to examine how the similarity in sound affected the results. Because the words used in the present study were limited to Japanese loanwords and their corresponding English words, some attributes of the words (e.g., the degree of frequency) used in these two studies could not be controlled. Therefore, the results of the present study could not be directly compared with those of Ikeda(1993). However, comparing the results of these studies would imply some suggestions to the effect of similarity in sound of English distracting words to Japanese words. In Experiment 1, the subjects were required to name the category of an English target word, ignoring a Japanese distracting word. It was predicted that inhibition and facilitation would be produced like in the task using ordinary words, because Japanese distracting words ignored are automatically processed. In Experiment 2, the subjects were required to name the category of a Japanese target word, ignoring an English distracting word. If an English distracting word which is similar to a Japanese word in sound is processed to affect the semantic processing of a Japanese target word like congnates as in de Groot and Nas (1991), the English word would produce inhibition and facilitation to a Japanese target word. If an English word is not processed from entry at all in spite of its similarity in sound to a Japanese word, the results in Experiment 2 would be similar to those in the task using ordinary words which were different in sound and script (Ikeda, 1993, Experiment 2). That is, neither inhibition nor facilitation would be produced.

To examine the time course of inhibition and facilitation, the SOA (stimulus onset asynchrony) between target and distractor was operated. The results about the SOA in the present study were compared with those in the task using ordinary words. 


\section{EXPERIMENT 1}

\section{Method}

Subjects: Ten subjects, 5 men, and 5 women, participated in this experiment. All of them were university students, and the average age was 20 years and 4 months. Their participation was voluntary. They were all native speakers of Japanese. Their visual acuities were normal or corrected with glasses. They had learned English as a foreign language since their entrances to junior high schools. They were assumed to be average Japanese university students in the proficiency in English.

Materials: Three members (items) from each of three categories were chosen; animals (KOALA, PANDA, LION), fruits (ORANGE, BANANA, MELON), and vehicle (BUS, BOAT, TRUCK). Each of these nine members (words) of the three categories was presented in English lower case words and Japanese Katakana words. The control stimuli were a row of $\times$ s. Japanese Katakana is phonetically based symbol like English alphabet. In this point, Japanese Katakana is very different from Japanese Kanji which is logographic. All of the Japanese words used in the present study were loanwords, and they were similar to English words in sound, although they were not completely the same. For example, “パンダ [panda]” is pronounced [panda] in Japanese.

Instrumentation: The stimuli were presented in dark-field technique like in the other Stroop tasks: The white words were presented on a black background of the CRT screen. A letter of the words and the control distractors was $0.6^{\circ}$ high $\times 0.6^{\circ}$ wide. The center of the stimuli was aligned $2^{\circ}$ above or below the fixation point, a cross $\left(1.7^{\circ} \times 1.7^{\circ}\right)$ on the CRT screen (NEC PC-KD854N). The reaction time was determined with an accuracy of $1 \mathrm{~ms}$ by means of voice key. Stimulus presentation and registration of reaction times were controlled by a personal computer (NEC PC-9801EX). The room was dimly lit. The subject sat $50 \mathrm{~cm}$ from the front of the CRT screen. The reaction time, the time elapsing between the onset of the target and the subject's response was measured and recorded for each trial. The experiment was run under the program control by the personal computer. The program controlled the random sequence and random position of the stimuli (above or below the fixation point).

Experimental design: The two variables of this experiment were SOA (stimulus onset asynchrony) and the target-distractor pairing. Both variables were varied within the subjects. The first variable, SOA, was varied in eight levels, $\pm 300, \pm 200, \pm 100, \pm 50 \mathrm{~ms}$. At the negative SOA levels, distractor (a Japanese word) was preexposed before target (an English word) by the SOA level. At the positive SOA levels, distractor was postexposed after target by the SOA level. To each subject, the sequence of the SOA blocks was randomized. The present study operated as many SOA levels as Glaser and Glaser $(1982,1989)$, because these previous studies indicated that inhibition and facilitation were produced within such a wide SOA range. Facilitation was usually produced at negative SOA levels (e.g., $-300 \mathrm{~ms}$ ), and large inhibition was produced at small SOA levels (e.g., $\pm 50 \mathrm{~ms}$ ). The second variable was the target-distractor pairing, which was varied in four levels. That is, in the category-congruent condition, the category of target was the same as that of distractor, but they were incongruent with each other (e.g., koala-ライオン[lion]). In the category-incongruent condition, the category of target was different from that of distractor, and they were incongruent with each other (e.g., koala-バス[bus]). In the congruent condition, target and distractor represented the same concept (e.g., koala-コアラ[koala]). In the control condition, target was accompanied with a nonword control stimulus (e.g., koala $-\times \times \times$ ). There were eight SOA blocks in the experiment. In each block, the SOA level was constant. The pairing was varied randomly from trial to trial within each SOA block. Each SOA block had 36 experimental trials, and each target was presented four times as target at the four pairing levels in one SOA block ( 9 targets $\times 4$ pairing levels $=36$ trials $)$.

Procedure: In advance, the subjects were shown all the English and Japanese words to be used as targets and distractors, and the names of the categories of those words. They were instructed to name the category of target (an English word) presented on the screen as fast as possible without error, ignoring a distracting word (a Japanese word). Each experimental block began with five training trials, in which target and distractor were presented at the same SOA level as that in the following experimental trials. The stimuli used in the training trials were different from those in the experimental trials. Before each SOA block, subjects were instructed that the target in that block was the first or the second one of the two stimuli presented on the screen. One experimental trial proceeded as follows: A cross was presented as a fixation point on the center of the screen for $1000 \mathrm{~ms}$; After the cross disappeared, target (or distractor) was presented above or below the cross; According to the SOA level, distractor (or target) was postexposed on the position 
Table 1. Reaction Time (ms) and Error Percentage (\%) in Each SOA and Pairing Condition in Experiment 1

\begin{tabular}{|c|c|c|c|c|c|c|c|c|c|}
\hline & \multicolumn{9}{|c|}{ Word categorizing task (target: English words-distractor: Japanese words) } \\
\hline & SOA (ms) & -300 & -200 & -100 & -50 & +50 & +100 & +200 & +300 \\
\hline \multicolumn{10}{|c|}{ Category-congruent } \\
\hline & Mean (ms) & 699 & 749 & 752 & 774 & 810 & 745 & 785 & 741 \\
\hline & $S D$ & 100 & 177 & 138 & 108 & 108 & 124 & 149 & 98 \\
\hline & Error $(\%)$ & 0 & 1 & 2 & 2 & 0 & 1 & 0 & 2 \\
\hline \multicolumn{10}{|c|}{ Category-incongruent } \\
\hline & Mean (ms) & 741 & 761 & 815 & 817 & 889 & 832 & 752 & 751 \\
\hline & $S D$ & 72 & 147 & 173 & 121 & 163 & 122 & 148 & 98 \\
\hline & Error $(\%)$ & 0 & 0 & 2 & 3 & 1 & 3 & 0 & 2 \\
\hline \multicolumn{10}{|l|}{ Control } \\
\hline & Mean (ms) & 732 & 717 & 773 & 769 & 787 & 770 & 764 & 764 \\
\hline & $S D$ & 82 & 126 & 131 & 127 & 119 & 121 & 126 & 103 \\
\hline & Error (\%) & 0 & 2 & 1 & 0 & 1 & 2 & 1 & 0 \\
\hline \multicolumn{10}{|c|}{ Congruent } \\
\hline & Mean (ms) & 709 & 735 & 713 & 772 & 817 & 756 & 773 & 762 \\
\hline & $S D$ & 131 & 158 & 122 & 140 & 176 & 123 & 166 & 113 \\
\hline & Error (\%) & 0 & 4 & 1 & 3 & 0 & 2 & 0 & 0 \\
\hline
\end{tabular}

opposite to the target (or the distractor). Both the target and the distractor disappeared at the same time when the subject responded to the target. Two thousands ms after the response, the cross was presented again on the center of the screen for the next trial. The experimenter recorded error trials. The experiment lasted about fifty minutes per subject.

\section{Results}

For each subject, the mean reaction time, standard deviation, and error rate were computed in SOA $\times$ target-distractor pairing conditions. The reaction time that deviated by more than two standard deviations from the mean in each condition were excluded. Table 1 shows the mean reaction time, standard deviation and error rate in each condition. Then the mean inhibition and facilitation were calculated (Fig. 1). As in the previous Stroop experiments, inhibition and facilitation were computed by subtracting the mean reaction time in the control condition from that in the category-congruent condition, in the category-incongruent condition, and in the congruent condition. Therefore, facilitation appeared as negative scores, while inhibition appeared as positive scores.

The errors were rare as in other Stroop tasks, so they were not subjected to any statistical analysis.

The complete set of data on the reaction time was subjected to a $8 \times 4$ (SOA $\times$ targetdistractor pairing) ANOVA with repeated measures within the subjects. The results 


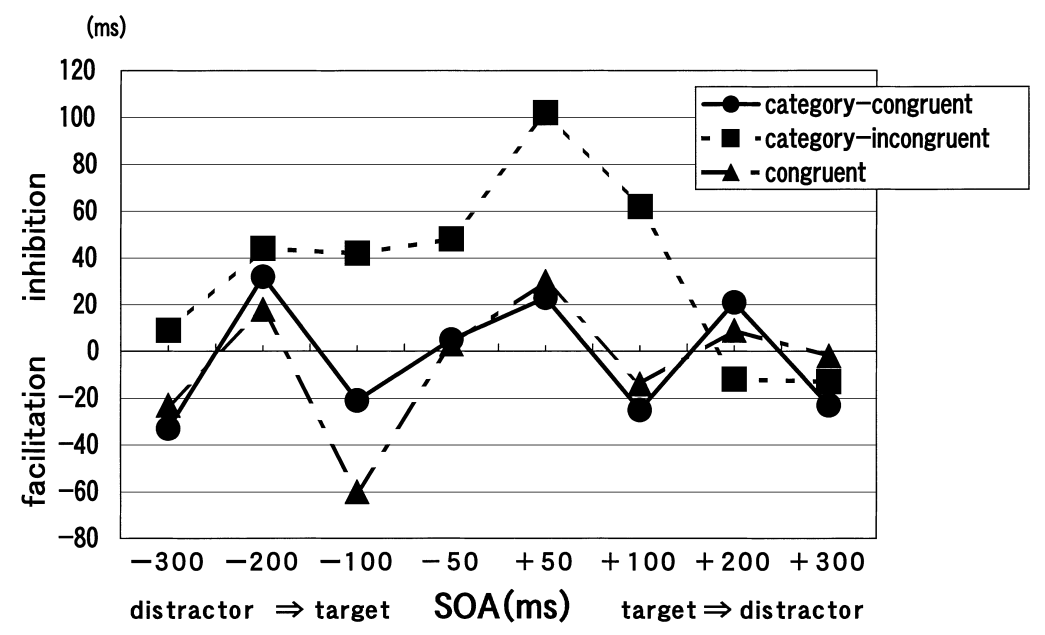

Fig. 1. Inhibition and facilitation in Experiment 1.

showed that the main effect of SOA $(F(7,63)=3.58, p<.005)$, the main effect of the targetdistractor pairing $(F(3,27)=6.86, p<.005)$, and the interaction between SOA and targetdistractor pairing $(F(21,189)=1.76, p<.05)$ were significant.

Multiple comparisons with Ryan's method (at the significance level of $p<.05$ ) were performed to examine the differences between eight SOA conditions. The results indicated that the the differences were significant between the SOA=-300 ms and the $\mathrm{SOA}=+50 \mathrm{~ms}$, and between the $\mathrm{SOA}=-200 \mathrm{~ms}$ and the $\mathrm{SOA}=+50 \mathrm{~ms}$. The mean reaction time at the $\mathrm{SOA}=+50 \mathrm{~ms}$ was the longest of all SOA conditions, though it was not statistically significant.

The main effect of the target-distractor pairing was based on the mean reaction time in each target-distractor pairing condition; $795 \mathrm{~ms}$ in the category-incongruent condition, $757 \mathrm{~ms}$ in the category-congruent condition, $755 \mathrm{~ms}$ in the congruent condition, and 760 $\mathrm{ms}$ in the control condition. Multiple comparisons with Ryan's method (at the significance level of $p<.05$ ) were performed to examine the differences between these mean reaction times. The results indicated that the differences among the target-distractor pairing conditions were significant between the category-incongruent condition and the control condition, and between the category-congruent condition and the categoryincongruent condition. Inhibition was produced only in the category-incongruent condition, and the significant difference between the reaction time in the categorycongruent condition and that in the category-incongruent condition indicated the effect of semantic relation between target and distractor.

Multiple comparisons with Ryan's method (at the significance level of $p<.05$ ) were performed to examine the interaction between SOA and target-distractor pairing. The results indicated that the differences between the category-incongruent condition and the control condition, and between the category-congruent condition and the categoryincongruent condition were significant only at the $\mathrm{SOA}=+50 \mathrm{~ms}$ and the $\mathrm{SOA}=+100 \mathrm{~ms}$. 
Table 2. Reaction Time (ms) and Error Percentage (\%) in Each SOA and Pairing Condition in Experiment 2

\begin{tabular}{|c|c|c|c|c|c|c|c|c|c|}
\hline & \multicolumn{9}{|c|}{ Word categorizing task (target: Japanese words-distractor: English words) } \\
\hline & SOA (ms) & -300 & -200 & -100 & -50 & +50 & +100 & +200 & +300 \\
\hline \multicolumn{10}{|c|}{ Category-congruent } \\
\hline & Mean (ms) & 645 & 668 & 670 & 693 & 759 & 706 & 683 & 671 \\
\hline & $S D$ & 97 & 143 & 126 & 143 & 243 & 163 & 165 & 139 \\
\hline & Error $(\%)$ & 1 & 1 & 0 & 3 & 2 & 1 & 1 & 1 \\
\hline \multicolumn{10}{|c|}{ Category-incongruent } \\
\hline & Mean (ms) & 669 & 677 & 703 & 734 & 745 & 685 & 674 & 680 \\
\hline & $S D$ & 118 & 139 & 181 & 165 & 196 & 139 & 167 & 186 \\
\hline & Error (\%) & 4 & 2 & 6 & 3 & 2 & 1 & 0 & 3 \\
\hline \multicolumn{10}{|l|}{ Control } \\
\hline & Mean (ms) & 620 & 667 & 654 & 682 & 689 & 707 & 669 & 668 \\
\hline & $S D$ & 106 & 122 & 132 & 126 & 166 & 160 & 136 & 169 \\
\hline & Error (\%) & 0 & 1 & 2 & 4 & 4 & 3 & 1 & 1 \\
\hline \multicolumn{10}{|c|}{ Congruent } \\
\hline & Mean (ms) & 655 & 618 & 640 & 662 & 742 & 688 & 654 & 670 \\
\hline & $S D$ & 129 & 123 & 124 & 115 & 231 & 139 & 115 & 137 \\
\hline & Error (\%) & 1 & 3 & 2 & 0 & 1 & 1 & 0 & 1 \\
\hline
\end{tabular}

\section{EXPERIMENT 2}

\section{Method}

Subjects: Twelve subjects, 10 men, and 2 women, participated in this experiment. They were similar to those in Experiment 1. The average age was 21 years and 2 months. None of them participated in Experiment 1.

Materials: The nine English words and the corresponding nine Japanese words used in Experiment 1 were also used in Experiment 2. However, in Experiment 2, Japanese words were presented as targets and English words were presented as distractors.

Instrumentation and experimental design: Instrumentation and experimental design were similar to those in Experiment 1.

Procedure: The procedure was mostly similar to that of Experiment 1 . The subjects were instructed to name the category of a Japanese target word, ignoring an English distracting word.

\section{Results}

For each subject, the mean reaction time, standard deviation, and error rate were computed in SOA $\times$ target-distractor pairing conditions. The reaction time that deviated by more than two standard deviations from the mean in each condition were excluded. Table 2 shows the mean reaction time, standard deviation and error rate in each condition. Then the mean inhibition and facilitation were calculated (Fig. 2). 


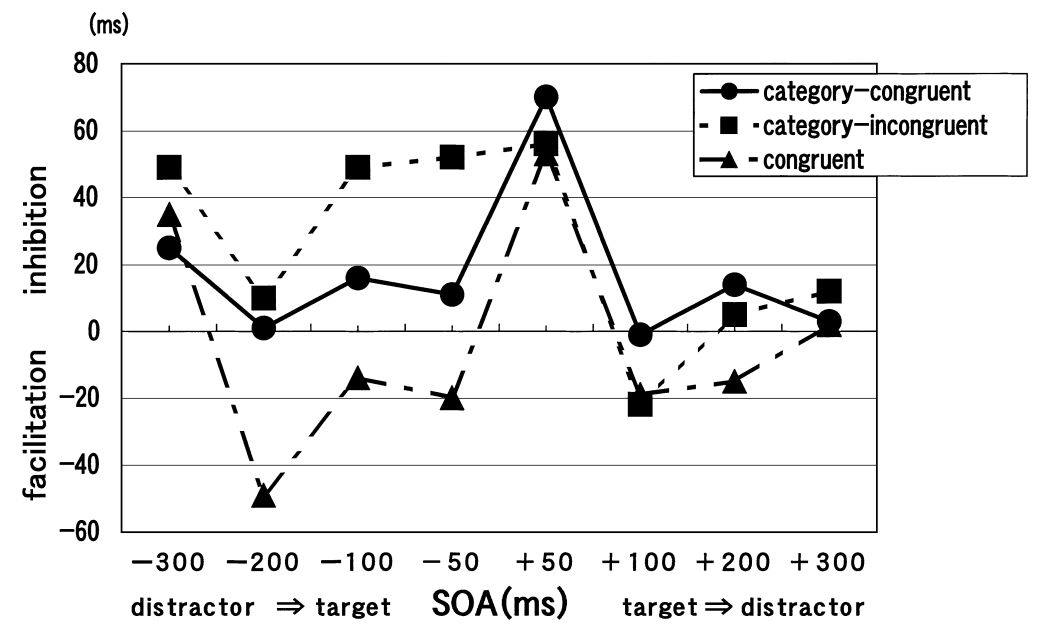

Fig. 2. Inhibition and facilitation in Experiment 2.

The errors were not subjected to any statistical analysis like in Experiment 1.

The complete set of data on the reaction time was subjected to a $8 \times 4(\mathrm{SOA} \times$ targetdistractor pairing) ANOVA with repeated measures within the subjects. The results showed that the main effect of SOA $(F(7,77)=2.55, p<.05)$ and the main effect of the target-distractor pairing $(F(3,33)=5.14, p<.01)$ were significant. The interaction between SOA and target-distractor pairing was not significant.

Multiple comparisons with Ryan's method (at the significance level of $p<.05$ ) were performed to examine the differences between eight SOA conditions. The results indicated that the the difference was significant between the SOA=-300 $\mathrm{ms}$ and the $\mathrm{SOA}=+50 \mathrm{~ms}$. The mean reaction time at the $\mathrm{SOA}=+50 \mathrm{~ms}$ was the longest of all SOA conditions, though it was not statistically significant.

The main effect of the target-distractor pairing was based on the mean reaction time in each target-distractor pairing condition; $696 \mathrm{~ms}$ in the category-incongruent condition, $687 \mathrm{~ms}$ in the category-congruent condition, $666 \mathrm{~ms}$ in the congruent condition, and 670 $\mathrm{ms}$ in the control condition. Multiple comparisons with Ryan's method (at the significance level of $p<.05$ ) were performed to examine the differences between these mean reaction times. The results indicated that the differences among the target-distractor pairing conditions were significant between the category-incongruent condition and the control condition. That is to say, inhibition was produced only in the categoryincongruent condition.

\section{GENERAL DisCuSSION}

The present study examined inhibition and facilitation between the English and the Japanese loanwords. The results of the present study showed that inhibition was produced 
when the target was an English word and the distractor was a Japanese word (Experiment 1), and when the target was a Japanese word and the distractor was an English word (Experiment 2). It was notable that inhibition was produced by an English distracting word when the target word was Japanese. This inhibition was not found in a similar target-distractor pairing task using ordinary words (Ikeda, 1993). It was assumed that the similarity of an English distracting word to a Japanese word in sound might affect this inhibition.

The inhibition and facilitation in such a task have been explained as follows: When a target word was processed, a distracting word was also processed in the same direction (Glaser \& Glaser, 1989). For example, when a target word was categorized, a distracting word was processed to be categorized, and inhibition and facilitation to a target word were produced. However, ordinary English distracting words were not assumably processed to be categorized, therefore, they did not produce inhibition and facilitation to Japanese target words (Ikeda, 1993).

Why did an English distracting word which was similar in sound to a Japanese word produce inhibition to a Japanese target word? It was assumed that English distracting words ignored might be processed from entry at least until phonetic processing in spite of its alphabetic script, the English word corresponding to a Japanese loanword would access the same lexical representation based on sound as the corresponding Japanese word, and it would automatically continue to be processed from the lexicon to the semantic memory like a Japanese word. Therefore, an English distracting word would produce inhibition to a Japanese target word to be categorized. On the other hand, the ordinary English word would not access the same lexical representation as the corresponding Japanese word, and would not continue to be processed to the semantic memory. Therefore, it would not produce inhibition. These results suggested the possibility that English words are automatically phonetically processed. If the English word was not processed at all, inhibition would not be produced even when it was similar to a Japanese word in sound.

The results of the present study implied a suggestion to the question indicated by de Groot and Nas (1991). In de Groot and Nas (1991), only cognates primed each other between languages like within languages. From these results, de Groot and Nas (1991) implied the question why similarity between the two languages in sound and spelling enhanced the semantic interaction in the common semantic memory. The answer to this question was suggested by the explanation described above. It is assumed that the secondlanguage words which were similar in both sound and spelling to the first-language words would access the common lexical representation to them, and it would be automatically processed from the lexicon to the semantic memory. Therefore, the semantic interaction between the two languages was easily produced.

Though facilitation was exhibited in the interlingual similar task in which target was an English word and distractor was a Japanese word (Ikeda, 1993), facilitation was not exhibited both in Experiment 1 and in Experiment 2 in the present study. Some previous studies (Glaser \& Glaser, 1982; Ikeda, 1995) reported that facilitation in the intralingual similar tasks disappeared unexpectedly. It also must await future researches to clarify the disappearance of facilitation in the present study. 
One of the purposes of the present study was to compare the results of the SOA in the present study with those in Ikeda (1993) which used ordinary words. The characteristic pattern of the SOA in the present study was that the usual increase of the reaction time at the short SOA like at the $\mathrm{SOA}=+50 \mathrm{~ms}$ was exhibited again. This increase of the reaction time at the short SOA was usually exhibited in the intralingual word-word categorizing task (e.g., both of target and distractor were Japanese, Ikeda, 1995). However, it was not exhibited in the interlingual word-word categorizing task (e.g., target was English and distractor was Japanese, Ikeda, 1993, Experiment 1). In Ikeda (1993), W. R. Glaser commented on the diasppearance of this peak in the interlingual task. It might be due to the fact that the subjects could discriminate the target stimulus and the distracting stimulus easily even at such a short SOA, because the target and distractor were represented in different form (e.g., English word and Japanese word). Therefore, the peak of the reaction time at the short SOA caused by the difficulty in discriminating between target and distractor was not exhibited in the interlingual task. However, this peak of the reaction time was exhibited again in the interlingual tasks in Experiments 1 and 2 of the present study. It can be argued as follows: The Japanese words and the English words were very similar in sound in the present study, though they were quite different in script. This similarity in sound caused the difficulty again in discriminating between the target stimulus and the distracting stimulus like in the ordinary intralingual tasks.

In Glaser and Glaser (1989) and Experiment 1 of the present study, the interaction between SOA and target-distractor pairing was significant in the word-word categorizing task, and a large inhibition was produced in the category-incongruent condition at small SOAs. Glaser and Glaser (1989) called this inhibition as a fast inhibition. The lack of the interaction in Experiment 2 of the present study suggested that the inhibition was produced more slowly. It might be due to the slower processing of the English distractors than the Japanese ones, though further research will be necessary to answer the question.

The results of the present study suggested the possibility that English words ignored are automatically phonetically processed by Japanese university students. It is necessary to examine to what extent they are processed in more proficient or less proficient subjects in English.

\section{REFERENCES}

Caramazza, A., \& Brones, I. 1980. Semantic classification by bilinguals. Canadian Journal of Psychology, 34, 77-81.

Chen, H.-C., \& Ng, M.-L. 1989. Semantic facilitation and translation priming effects in Chinese-English bilinguals. Memory \& Cognition, 17, 454-462.

Glaser, M. O., \& Glaser, W. R. 1982. Time course analysis of the Stroop phenomenon. Journal of Experimental Psychology: Human Perception and Performance, 8, 875-894.

Glaser, W. R., \& Glaser, M. O. 1989. Context effects in Stroop-like word and picture processing. Journal of Experimental Psychology: General, 118, 13-42.

de Groot, A. M. B., \& Nas, G. L. J. 1991. Lexical representation of cognates and noncognates in compound bilinguals. Journal of Memory and Language, 30, 90-123.

Guttentag, R. E., Haith, M. M., Goodman, G. S., \& Hauch, J. 1984. Semantic processing of unattended words 
by bilinguals: A test of the input switch mechanism. Journal of Verbal Learning and Verbal Behavior, 23, 178-188.

Ikeda, S. 1993. Semantic interaction between Japanese and English words: Interlingual inhibition and facilitation in the word-word categorizing task. Hiroshima Forum for Psychology, 15, 17-27.

Ikeda, S. 1995. Interference observed in the word categorizing task. Japanese Journal of Psychonomic Science, 13, 93-96. (in Japanese with English abstract)

Potter, M. C., So, K. -F., Von Eckardt, B., \& Feldman, L. B. 1984. Lexical and conceptual representation in beginning and proficient bilinguals. Journal of Verbal Learning and Verbal Behavior, 23, 23-38.

Snodgrass, J. G. 1984. Concepts and their surface representations. Journal of Verbal Learning and Verbal Behavior, 23, 3-22.

(Manuscript received October 1, 2003; Revision accepted February 17, 2004) 